\title{
Bounds on dissipation in stress-driven flow in a rotating frame
}

\author{
By W. TAN G ${ }^{1}$, C. P. CAULFIELD ${ }^{1} \dagger$ AND W. R. YOUNG \\ ${ }^{1}$ Department of Mechanical and Aerospace Engineering, Jacobs School of Engineering, \\ University of California, San Diego, 9500 Gilman Drive, La Jolla, CA 92093-0411, USA \\ ${ }^{2}$ Scripps Instititution of Oceanography, University of California, San Diego, 9500 Gilman Drive, \\ La Jolla, CA 92093-0213, USA
}

(Received 9 December 2004 and in revised form 4 April 2005)

We calculate a rigorous dual bound on the long-time-averaged mechanical energy dissipation rate $\varepsilon$ within a channel of an incompressible viscous fluid of constant kinematic viscosity $v$, depth $h$ and rotation rate $f$, driven by a constant surface stress $\boldsymbol{\tau}=\rho u_{\star}^{2} \hat{\imath}$, where $u_{\star}$ is the friction velocity. It is well known that $\varepsilon \leqslant \varepsilon_{\text {Stokes }}=u_{\star}^{4} / \nu$, i.e. the dissipation is bounded above by the dissipation associated with the Stokes flow.

Using an approach similar to the variational 'background method' (due to Constantin, Doering \& Hopf), we generate a rigorous dual bound, subject to the constraints of total power balance and mean horizontal momentum balance, in the inviscid limit $\nu \rightarrow 0$ for fixed values of the friction Rossby number $R o_{\star}=u_{\star} /(f h)=$ $\sqrt{G} E$, where $G=\tau h^{2} /\left(\rho v^{2}\right)$ is the Grashof number, and $E=v / f h^{2}$ is the Ekman number. By assuming that the horizontal dimensions are much larger than the vertical dimension of the channel, and restricting our attention to particular, analytically tractable, classes of Lagrange multipliers imposing mean horizontal momentum balance analogous to the ones used in Tang, Caulfield \& Young (2004), we show that $\varepsilon \leqslant \varepsilon_{\max }=$ $u_{\star}^{4} / v-2.93 u_{\star}^{2} f$, an improved upper bound from the Stokes dissipation, and $\varepsilon \geqslant \varepsilon_{\min }=$ $2.795 u_{\star}^{3} / h$, a lower bound which is independent of the kinematic viscosity $v$.

\section{Introduction}

Forced turbulent flows are common in the natural environment. One way to characterize such turbulent motion is through identifying scaling laws for the mechanical energy dissipation rate, which capture how turbulent small-scale motions dissipate the energy input by the large-scale external forcing. It is quite natural that properties of the mechanical energy dissipation rate are strongly affected by different types of forcings. In Tang, Caulfield \& Young (2004, henceforth TCY04) we investigated the specific case where stress is applied at the upper surface of a layer of fluid as happens, for example, when wind blows over a lake. In TCY04, we considered the stress-driven flow in a non-rotating frame and found that the mechanical energy dissipation rate is bounded above by the laminar flow $\varepsilon \leqslant u_{\star}^{4} / \nu$, and below by $\varepsilon \geqslant 7.531 u_{\star}^{3} / h$, where the mechanical energy dissipation rate is defined as

$$
\varepsilon=\frac{\nu^{3}}{h^{4}}\left\langle\|\nabla \boldsymbol{u}\|^{2}\right\rangle .
$$

$\dagger$ Present address: BP Institute for Multiphase Flow and Department of Applied Mathematics and Theoretical Physics, University of Cambridge, UK. 
In $(1.1)$

$$
\|\nabla \boldsymbol{u}\|^{2} \equiv|\nabla u|^{2}+|\nabla v|^{2}+|\nabla w|^{2},
$$

is the non-dimensional deformation (using the kinematic viscosity $v$ and the constant layer depth $h$ as characteristic scales) and $\langle\cdot\rangle$ is a spatial and temporal average defined in more detail below. This result is satisfying for small-scale problems because the lower bound is independent of molecular viscosity. However, when the flow domain is significantly larger, we would also expect rotation to play an important role, and this paper is dedicated to studying such contributions. The effects of rotation have been considered for the problem of generating bounds on heat transport in rotating convection (Constantin, Hallstrom \& Putkaradze 1999, 2001; Vitanov \& Busse 2001; Vitanov 2001, 2003; Yan 2004), but not previously in the context of dissipation within stress-driven flows.

In this paper, we use an approach with points of similarity to the variational method developed by Constantin and Doering utilizing a mathematical device introduced by Hopf (1941) to construct bounds on the mechanical energy dissipation rate (Doering \& Constantin 1992, 1994; Constantin \& Doering 1995; Doering \& Constantin 1996; Nicodemus, Grossmann \& Holthaus 1997a,b, 1998a,b). Following Plasting \& Kerswell (2003, henceforth PK03), we refer to the original method as the CDH method. The $\mathrm{CDH}$ method is based around a non-unique decomposition of the flow fields into a 'background' which satisfies the boundary conditions, and a 'fluctuation' away from this background which has homogeneous boundary conditions. Variational methods may then be used (through the derivation of Euler-Lagrange equations) to generate bounds on (for example) the mechanical energy dissipation rate, subject to physically motivated imposed constraints, such as total energy balance, and horizontally averaged momentum balances. As discussed in PK03, under the plausible though unproven assumption of streamwise invariance, solutions to the Euler-Lagrange equations can in principle be identified using numerical continuation techniques to asymptotically large Reynolds numbers, which allow the identification of characteristic scalings.

However, the particular problem which we are considering has several complications compared to previous work, which make use of the $\mathrm{CDH}$ method somewhat problematic. As discussed below in more detail, since the system is rotating, it is important to apply constraints on horizontal averages of momentum both parallel and perpendicular to the direction of application of the surface stress. Also, since we are interested in the effect of both stress and rotation on the flow evolution of a viscous fluid, there are two non-dimensional control parameters, e.g. an Ekman number and a Grashof number. Furthermore, due to the effect of the rotation, the rotating laminar solution is non-trivially different from a simple linear velocity profile, which also has implications for the generation of simple bounds on the flow dissipation. Because of the complexities introduced by these aspects of the flow under consideration, in this paper, we propose a related method, which still imposes the physically important constraints of horizontally averaged momentum, but uses a somewhat different approach.

The critical characteristic which this method shares with the CDH method is the flexibility in the vertical structure of the Lagrange multiplier which imposes the two horizontal averages of momentum. Using mathematically tractable trial functions for this Lagrange multiplier simplifies the analysis significantly compared to the numerical methods presented by PK03, and allows us to treat the bifurcation away from the rotating laminar solution in a natural way, as the relevant controlling parameters are varied. For the non-rotating stress-driven flow considered in TCY04, 
this method was shown to yield the same dimensional scaling for the lower bound on the dissipation as the full $\mathrm{CDH}$ method, although for the particular trial functions tested the premultiplying numerical factor on the scaling was underestimated by $34 \%$ as the kinematic viscosity $v \rightarrow 0$.

Indeed, we can generate both a lower bound and an upper bound using trial functions within our proposed framework. Interestingly, unlike the non-rotating flow discussed in TCY04, the rotating laminar solution ceases to be relevant as either an upper or lower bound for sufficiently strong forcing within this framework. As in the CDH method, we ensure the validity of the bounds by requiring that the trial functions satisfy a 'spectral constraint'. The spectral constraint is essentially a statement of the energy stability (i.e. the asymptotic global stability of a flow to arbitrary disturbances in the sense that the disturbance energy decays to zero monotonically as $t \rightarrow \infty$ (see for example Joseph 1976). A (notional) flow with mean profile given by the Lagrange multiplier imposing a momentum balance determined by a solution set of the EulerLagrange equations is marginally energy stable if and only if that specific solution set corresponds to a bound. Just as in the $\mathrm{CDH}$ method, our method leads to a spectral constraint, quadratic in perturbation quantities, that corresponds to verifying that the notional flow with mean profiles determined by the Lagrange multipliers is marginally energy stable. The asymptotic scalings of the bounds may then be found by fixing the external stress and rotation rate, while lowering the flow viscosity $v$ to the inviscid limit.

The rest of the paper is organized as follows. In $\S 2$, we introduce the governing equations and formulate the stress-driven flow model. In $\S 3$, we obtain the laminar solution to the rotating problem, and study the energy stability of the laminar solution. In $\S 4$, we obtain a dual bound on the mechanical energy dissipation rate subject to the constraints of total power balance and mean horizontal momentum balance in two perpendicular directions. In $\S 5$, we construct the dual bound using a special family of trial functions that satisfy the same governing equations as the laminar solutions (which we refer to as the 'pseudo-laminar profiles'). Once the rotating laminar solution loses energy stability, we find that it is neither an upper nor a lower bound within our framework. Finally, in $\S 6$, we draw some conclusions, in particular illustrating points of similarity and difference between our method and the CDH method.

\section{Formulation}

We consider an incompressible layer of fluid confined in a zonal channel with constant depth $h$, density $\rho$, and kinematic viscosity $v$. With geophysical applications in mind, we use the Coriolis parameter, $f$, which is related to the planetary rotation rate, $\Omega$, by $f=2 \Omega \sin \theta$, where $\theta$ is the latitude (Gill 1982; Pedlosky 1987). Motion is driven by a uniform stress $\boldsymbol{\tau}=\rho u_{\star}^{2} \hat{\boldsymbol{\imath}}$ applied at the upper top surface, $z=0$. The friction velocity is $u_{\star}$ and $\hat{\boldsymbol{\imath}}$ is a unit vector in the zonal $(x)$ direction. At the bottom, $z=-h$, there is a no-slip boundary condition. The Navier-Stokes equations can then be scaled using $\rho, v$, and $h$ to define units of mass, time and length. The resulting governing equations are

$$
\begin{aligned}
\boldsymbol{u}_{t}+\boldsymbol{u} \cdot \nabla \boldsymbol{u}+\nabla p+E^{-1} \hat{\boldsymbol{k}} \times \boldsymbol{u} & =\nabla^{2} \boldsymbol{u}, \\
\nabla \cdot \boldsymbol{u} & =0,
\end{aligned}
$$

with boundary conditions

$$
z=0: \quad u_{z}=G, \quad v_{z}=0, \quad w=0 ; \quad z=-1: \quad \boldsymbol{u}=0 .
$$




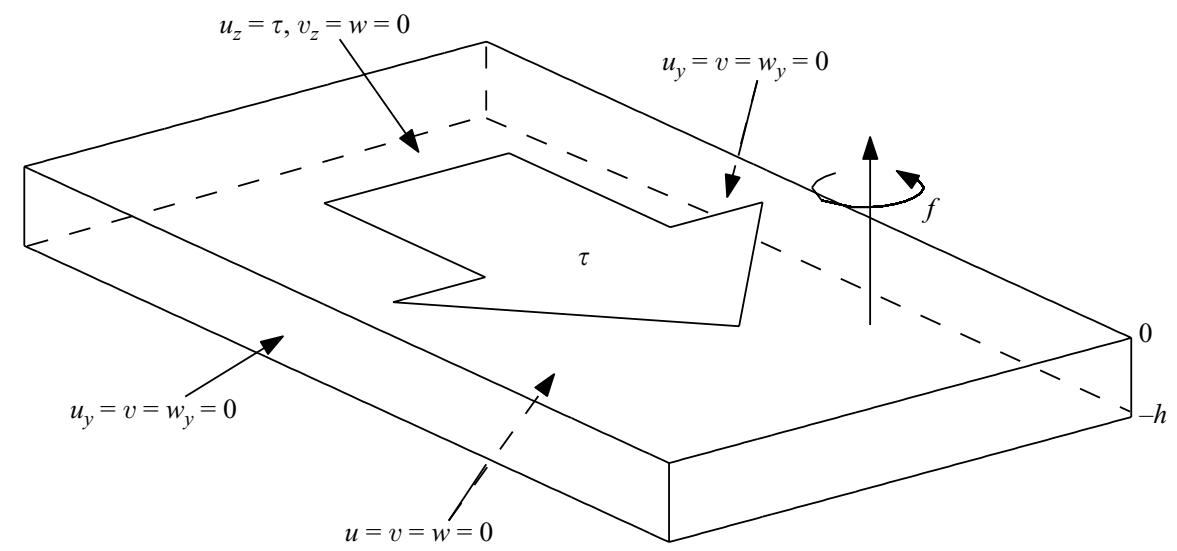

FiguRE 1. Schematic figure of the flow geometry, showing the coordinate system, boundary conditions and rotation axis. The stress $\tau$ acts in the zonal $(x)$ direction. Because the layer is shallow $\left(h / L_{x}\right.$ and $h / L_{y}$ are very small) only the vertical component of the Coriolis force is retained.

The important non-dimensional parameters used above are the Grashof number $G$, and the Ekman number $E$, defined as

$$
G \equiv \frac{\tau h^{2}}{\rho v^{2}}=\frac{u_{\star}^{2} h^{2}}{v^{2}}, \quad E \equiv \frac{v}{f h^{2}} .
$$

We sometimes use a parameter, $R o_{\star}$, a 'friction' Rossby number, defined by

$$
R o_{\star}=\sqrt{G} E=\frac{u_{\star}}{f h} .
$$

Because $R o_{\star}$ is independent of $v$, the inviscid asymptotic limit is obtained by taking $G \rightarrow \infty$ with $R o_{\star}$ fixed. It is interesting to make a rough numerical estimate of $R o_{\star}$ using geophysical values. For example, if $u_{\star}=0.01 \mathrm{~m} \mathrm{~s}^{-1}, f=10^{-5} \mathrm{~s}^{-1}, v=10^{-6} \mathrm{~m}^{2} \mathrm{~s}^{-1}$ and $h=4000 \mathrm{~m}$, then $R o_{\star}=0.25$ and $G=O\left(10^{15}\right)$. Thus in the geophysical regime $R o_{\star}$ is a smallish number and $G$ is very large.

We are considering a re-entrant channel, so that in the zonal (or streamwise $x$ ) direction the flow is periodic, i.e. $\boldsymbol{u}(\boldsymbol{x}, t)=\boldsymbol{u}\left(\boldsymbol{x}+L_{x} \hat{\boldsymbol{\imath}}, t\right)$. In the meridional (or spanwise $y$ ) direction, the channel is bounded by impermeable stress-free walls at $y=0$ and $L_{y}$. Thus the important meridional boundary conditions are $v(x, 0, z, t)=v\left(x, L_{y}, z, t\right)=0$. This is a very simple idealization of the Antarctic Circumpolar Current: the flow is reentrant in the zonal direction and subject to a constraint that there is no net meridional mass flux (see the discussion surrounding (2.8)). The flow is shown schematically in figure 1.

We define a mean flow, $\bar{u}(z)$ and $\bar{v}(z)$, using a time and horizontal average:

$$
\bar{q}(z) \equiv \lim _{T \rightarrow \infty} \frac{1}{L_{y} L_{x} T} \int_{0}^{T} \int_{0}^{L_{y}} \int_{0}^{L_{x}} q(\boldsymbol{x}, t) \mathrm{d} x \mathrm{~d} y \mathrm{~d} t .
$$

We also need a further volume average:

$$
\langle q\rangle=\int_{-1}^{0} \bar{q}(z) \mathrm{d} z
$$

We characterize the flow using the mechanical energy dissipation per unit mass, $\varepsilon$, as defined in (1.1), using the volume average defined by (2.6). Using a well-known 
argument, reprised in TCY04, one can show that $\varepsilon$ is bounded from above by the dissipation produced by the non-rotating Stokes flow, i.e. the laminar solution in a non-rotating flow:

$$
\boldsymbol{s}=G(z+1) \hat{\boldsymbol{\imath}} ; \quad \varepsilon \leqslant \varepsilon_{S}=\frac{u_{\star}^{4}}{v}=\frac{v^{3}}{h^{4}} G^{2} .
$$

We seek opportunities to improve this upper bound, but our main goal is to find a lower bound on $\varepsilon$.

With geophysical applications in mind, we are interested in a channel whose depth is much less than both horizontal dimensions, i.e. $L_{x}$ and $L_{y}$ are both much greater than unity. In this circumstance we might expect that the impact of the sidewalls at $y=0$ and $L_{y}$ is relatively minor. But associated with the sidewalls, there is a massconservation constraint that at every $y$

$$
\int_{0}^{L_{x}} \int_{-1}^{0} v(\boldsymbol{x}, t) \mathrm{d} z \mathrm{~d} x=0 .
$$

This condition is relevant to all real channel flows, and entails an undetermined pressure drop across the channel, supported by the rigid sidewalls at $y=0$ and $L_{y}$. Thus it is physically inconsistent to assume that pressure is a periodic function of $y-$ the pressure must have a linear component, $p \propto y$, to ensure that there is a net drop between $y=0$ and $L_{y}$. This pressure drop is the main agent through which distant sidewalls influence the flow in the middle of the channel. This issue has been ignored by applications of the $\mathrm{CDH}$ method, which tacitly assume that all dynamical variables are periodic functions of $x$ and $y$, so that for instance the horizontal average of $p_{y}$ is zero. In the non-rotating case this issue is probably unimportant because only the zonal $(x)$ momentum constraint is incorporated into the bound. However if rotation is important then it is essential to use both the $x$ and the $y$ momentum constraints. Incorporating the $y$ momentum constraint forces one to confront (2.8) and the associated unknown pressure drop.

\section{The rotating laminar solution and its energy stability}

In the middle of the channel, away from the sidewalls, the rotating laminar solution of the Navier-Stokes equations (2.1a) has the form

$$
\boldsymbol{u}=G U(z) \hat{\boldsymbol{\imath}}+G V(z) \hat{\boldsymbol{\jmath}} .
$$

This laminar solution inevitably varies from the non-rotating laminar Stokes flow $s$ defined in (2.7) due to the effect of the Coriolis force. The solution is obtained by solving

$$
\begin{aligned}
-V & =E U_{z z}, \\
U-U_{\mathrm{g}} & =E V_{z z},
\end{aligned}
$$

with the boundary conditions $U(-1)=V(-1)=0, U_{z}(0)=1$ and $V_{z}(0)=0$. The 'geostrophic velocity', $U_{\mathrm{g}} \equiv-E p_{y}$, is determined by requiring that

$$
\int_{-1}^{0} V(z) \mathrm{d} z=0
$$



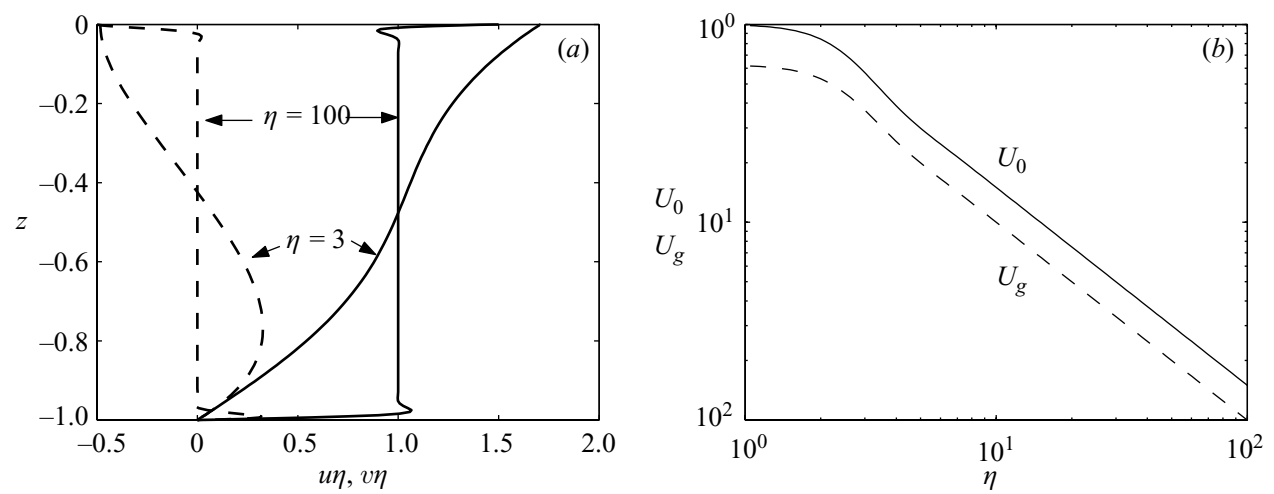

Figure 2. (a) The laminar solution to equations (3.2) for two values of $\eta \equiv(2 E)^{-1 / 2}$. The zonal $(x)$ velocity $U$ is plotted with solid lines, and the meridional $(y)$ velocity $V$ is plotted with dashed lines. Both velocities are scaled with $\eta$ so that they are $O(1)$ so that flows with different values of $\eta$ can be compared. The flow develops boundary layers when $\eta \gg 1$. $(b)$ The surface zonal velocity $U_{0}(\eta)$ (defined in (3.6) and plotted with a solid line) and geostrophic velocity $U_{\mathrm{g}}(\eta)$ (defined in (3.5), and plotted with a dashed line).

The uniform pressure gradient $U_{\mathrm{g}}$ produces the cross-channel pressure drop mentioned in the discussion surrounding $(2.8) . \dagger$

The solution of (3.2) and (3.3) is

$$
U+\mathrm{i} V=U_{\mathrm{g}}(\eta)\left\{1-\frac{\cosh (\sigma \eta z)}{\cosh (\sigma \eta)}+\frac{1}{\sigma \eta U_{\mathrm{g}}(\eta)} \frac{\sinh (\sigma \eta \bar{z})}{\cosh (\sigma \eta)}\right\},
$$

where $\sigma \equiv 1+\mathrm{i}, \bar{z} \equiv 1+z, \eta \equiv(2 E)^{-1 / 2}$, and

$$
U_{\mathrm{g}}(\eta) \equiv \frac{\cosh (2 \eta)+\cos (2 \eta)-2 \cosh (\eta) \cos (\eta)}{\eta[\sinh (2 \eta)-\sin (2 \eta)]}
$$

The zonal velocity at the surface is $U(0)=U_{0}(\eta)$, where

$$
U_{0}(\eta) \equiv \frac{3 \cosh (2 \eta)+2+3 \cos (2 \eta)-8 \cosh (\eta) \cos (\eta)}{2 \eta[\sinh (2 \eta)-\sin (2 \eta)]} .
$$

Figure 2(a) shows laminar velocity profiles at two values of $\eta$, while the functions $U_{\mathrm{g}}(\eta)$ and $U_{0}(\eta)$ are shown in figure $2(b)$.

\subsection{Rapid rotation $E \ll 1$}

In the rapidly rotating limit, with $\eta=(2 E)^{-1 / 2} \gg 1$, the interior solution is $(U, V)=$ $\left(U_{\mathrm{g}}, 0\right)$ and all the shear is confined to Ekman layers at $z=0$ and $z=-1$. In this case, the expressions above simplify to $U_{\mathrm{g}}(\eta) \approx 1 / \eta, U_{0}(\eta) \approx 3 / 2 \eta$ and

$$
\begin{aligned}
U & \approx \frac{1}{\eta}\left[1-\mathrm{e}^{-\eta \bar{z}} \cos \eta \bar{z}+\frac{1}{2} \mathrm{e}^{\eta z}(\cos \eta z+\sin \eta z)\right], \\
V & \approx \frac{1}{\eta}\left[\mathrm{e}^{-\eta \bar{z}} \sin \eta \bar{z}+\frac{1}{2} \mathrm{e}^{\eta z}(\sin \eta z-\cos \eta z)\right]
\end{aligned}
$$

where $\bar{z} \equiv 1+z$.

$\dagger$ In the non-rotating case the laminar solution is the Stokes flow in (2.7), with $p_{y}=p_{x}=0$. However if the flow is turbulent then the Reynolds stress $\overline{v^{\prime} w^{\prime}}$ might drive a $\bar{v}(z)$, which satisfies (2.8). Thus there is a cross-channel pressure drop in both the non-rotating and rotating cases. 


\subsection{The mechanical energy dissipation of the laminar solution}

The laminar dissipation is

$$
\varepsilon_{\mathrm{L}}=\frac{v^{3}}{h^{4}} G^{2} \times U_{0}(\eta)=\frac{u_{\star}^{4}}{v} \times U_{0}(\eta),
$$

where $U_{0}(\eta)$ is the non-dimensional function defined in (3.6). If $E \ll 1$ (strong rotation) then using $U_{0} \approx 3 /(2 \eta) \ll 1$, the expression above reduces to

$$
\varepsilon_{\mathrm{L}} \approx \frac{3 u_{\star}^{4}}{\sqrt{2 v f h}} .
$$

Notice that the laminar dissipation above is smaller than the Stokes dissipation defined in (2.7) by a factor of $\sqrt{E} \ll 1$ and, unlike in the non-rotating flow discussed in TCY04, the laminar dissipation is neither an upper nor a lower bound on the actual dissipation.

\subsection{Energy stability of the laminar solution}

Standard arguments (e.g. Drazin \& Reid 1981) show that the laminar solution (3.4) is globally asymptotically or energy stable (see Joseph 1976) provided that the Grashof number, $G$ in (2.3), is sufficiently small. Specifically, if

$$
G<G_{\mathrm{es}}(E)
$$

then disturbance energy decays monotonically. The energy stability eigenvalue, $G_{\mathrm{es}}(E)$, is defined by

$$
G_{\mathrm{es}}(E)=\min _{\boldsymbol{u}^{\prime}}\left(\frac{\left\langle\left\|\nabla \boldsymbol{u}^{\prime}\right\|^{2}\right\rangle}{\left\langle U_{z} u^{\prime} w^{\prime}+V_{z} v^{\prime} w^{\prime}\right\rangle}\right) .
$$

The minimum above is over the set of all incompressible velocities, $\boldsymbol{u}^{\prime}=\left(u^{\prime}, v^{\prime}, w^{\prime}\right)$, satisfying homogeneous boundary conditions corresponding to (2.2). Explicitly, the energy stability eigenvalue $G_{\text {es }}(E)$ is obtained as the smallest eigenvalue of the Orr problem:

$$
2 \nabla^{2} \boldsymbol{u}^{\prime}-G_{\mathrm{es}}\left(\begin{array}{c}
w^{\prime} \\
0 \\
u^{\prime}
\end{array}\right) U_{z}-G_{\mathrm{es}}\left(\begin{array}{c}
0 \\
w^{\prime} \\
v^{\prime}
\end{array}\right) V_{z}=\nabla p^{\prime}
$$

with the pressure $p^{\prime}$ determined so that $\nabla \cdot \boldsymbol{u}^{\prime}=0$.

We can solve this Orr problem by assuming that in the middle of the channel, the boundary conditions on the sidewalls are insignificant, and so expand the flow into Fourier modes in the horizontal directions and use Chebyshev polynomials in the vertical direction (see e.g. Boyd 2001): $\boldsymbol{u}^{\prime}(x, y, z)=\hat{\boldsymbol{u}}(z) \mathrm{e}^{\mathrm{i}(k x+l y)}, p^{\prime}(x, y, z)=$ $\hat{p}(z) \mathrm{e}^{\mathrm{i}(k x+l y)}$. The boundary conditions on the perturbation fields are

$$
\hat{\boldsymbol{u}}(-1)=0, \quad \hat{u}_{z}(0)=\hat{v}_{z}(0)=\hat{w}(0)=0 .
$$

Using D to denote $\partial / \partial z$, equation (3.12) becomes

$$
2\left(\mathrm{D}^{2}-k^{2}-l^{2}\right) \hat{\boldsymbol{u}}-G_{\mathrm{es}}\left(\begin{array}{c}
\hat{w} \\
0 \\
\hat{u}
\end{array}\right) U_{z}-G_{\mathrm{es}}\left(\begin{array}{c}
0 \\
\hat{w} \\
\hat{v}
\end{array}\right) V_{z}-\left(\begin{array}{c}
\mathrm{i} k \hat{p} \\
\mathrm{i} l \hat{p} \\
\mathrm{D} p
\end{array}\right)=0,
$$

and $G_{\mathrm{es}}$ is found corresponding to different $\eta$ by identifying the minimum value across possible choices of $k$ and $l$ in wavenumber space. We have found several energy stability points for different values of $\eta$ as shown in table 1, implying that 


\begin{tabular}{lllrll}
$\eta$ & $k$ & $l$ & $G_{\text {es }}\left(R o_{\star}\right)$ & \multicolumn{1}{c}{$R o_{\star}$} & \multicolumn{1}{c}{$E_{\text {es }}$} \\
0 & 0 & 2.08 & 51.73 & $\infty$ & $\infty$ \\
0.7071 & 0.11 & 2.08 & 51.88 & 7.2 & 1 \\
2.1213 & 1 & 1.87 & 63.19 & 0.88 & 0.11 \\
7.071 & 2.11 & 0.65 & 442.50 & 0.21 & 0.01 \\
21.213 & 2.99 & 0.58 & 3099.00 & 0.062 & 0.0011 \\
70.71 & 3.92 & 0.31 & 30291.22 & 0.017 & 0.0001 \\
212.13 & 4.69 & 0.15 & 261193.55 & 0.0057 & 0.000011
\end{tabular}

TABLE 1. Solutions of the eigenproblem (3.14) at the energy stability points, their dependence on the friction Rossby number $R o_{\star}$ defined in (2.4), and the corresponding $E_{\mathrm{es}}\left(R o_{\star}\right)=$ $R o_{\star} / \sqrt{G_{\mathrm{es}}\left(R o_{\star}\right)}$ associated with these points.
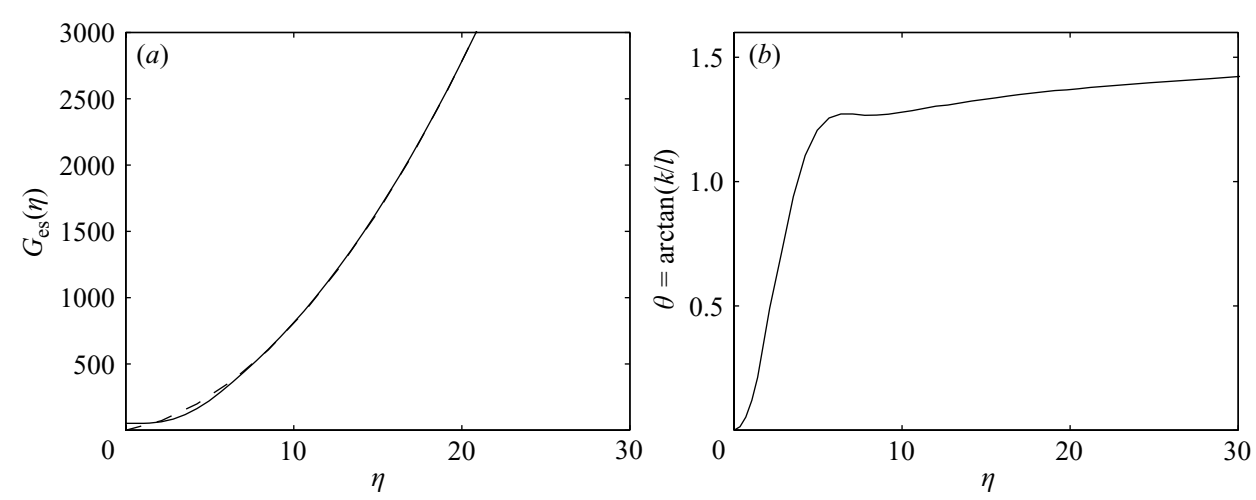

Figure 3. (a) The eigenvalue $G_{\text {es }}(\eta)$ obtained by solving the eigenproblem (3.14) (solid line) and the asymptotic approximation $G_{\mathrm{es}}(\eta) \approx 5.86 \eta^{2}+21.8 \eta$ (dashed line). $(b)$ The angle $\theta$ between the orientation of the horizontal rolls and the meridional direction. In the non-rotating limit $(\eta=0)$ the orientation is $\theta=0$ (completely meridional, with $k=0)$. As the rotation rate increases, and $\eta$ becomes large, $\theta \rightarrow \pi / 2$, and $l \rightarrow 0$.

asymptotically, $G_{\text {es }} \approx 5.86 \eta^{2}+21.8 \eta$. We show the general relationship between $G_{\text {es }}$ and $\eta$ in figure $3(a)$.

The orientation of the horizontal rolls associated with the most unstable perturbation changes with $\eta \equiv(2 E)^{-1 / 2}$. The non-rotating limit is $\eta=0$, and in this case $k$ is also zero; the roll axis is in the zonal $(x)$ direction and $u_{x}^{\prime}=p_{x}^{\prime}=0$. As $\eta$ increases, the roll axis rotates towards the meridional $(y)$ direction. In the rapidly rotating limit $\eta \rightarrow \infty$, the axes of the rolls are parallel with the meridional $(y)$ direction and $v_{y}^{\prime}=p_{y}^{\prime}=0$. The orientation of the rolls as a function of $\eta$ is shown in figure $3(b)$.

\section{Upper and lower bounds}

In this section we obtain complementary upper and lower bounds on the mechanical energy dissipation rate $\varepsilon$ subject to the integral constraints of total power balance and global horizontal momentum balance in both the $x$ - and $y$-directions using the technique discussed in TCY04. Although the basic idea is similar to the CDH method, the procedure is simpler than that required by the $\mathrm{CDH}$ method to generate the full bound. 
We begin by decomposing the flow velocity into a mean $(\bar{u}, \bar{v})$, plus a perturbation $\boldsymbol{u}^{\prime}$ so that the (non-dimensional) dissipation satisfies

$$
\left\langle\|\nabla \boldsymbol{u}\|^{2}\right\rangle-\left\langle\bar{u}_{z}^{2}\right\rangle-\left\langle\bar{v}_{z}^{2}\right\rangle-\left\langle\left\|\nabla \boldsymbol{u}^{\prime}\right\|^{2}\right\rangle=0 .
$$

The global energy integral is obtained by dotting $\boldsymbol{u}$ into the momentum equation (2.1a) and volume averaging. The result can be written as

$$
\left\langle\|\nabla \boldsymbol{u}\|^{2}\right\rangle-G\left\langle\bar{u}_{z}\right\rangle=0 \text {. }
$$

It is important to note that rotation plays no role in this global expression. The second term on the left-hand side is the (non-dimensional) surface stress, quantified by the Grashof number $G$, as defined in (2.3), times the average surface velocity, given by $\overline{u(x, y, 0, t)}=\left\langle u_{z}\right\rangle$.

Horizontally averaging the horizontal components of the momentum equation $(2.1 a)$ yields the constraints

$$
\begin{aligned}
\left(\overline{u^{\prime} w^{\prime}}\right)_{z}-\bar{u}_{z z}-E^{-1} \bar{v} & =0 \\
\left(\overline{v^{\prime} w^{\prime}}\right)_{z}-\bar{v}_{z z}+E^{-1} \bar{u}-E^{-1} u_{\mathrm{g}} & =0
\end{aligned}
$$

where the 'geostrophic velocity', $u_{\mathrm{g}}$, is

$$
u_{\mathrm{g}} \equiv-E^{-1} \overline{p_{y}}=-\lim _{T \rightarrow \infty} \frac{E^{-1}}{L_{y} L_{x} T} \int_{0}^{T} \int_{0}^{L_{x}}[p]_{y=0}^{y=L_{y}} \mathrm{~d} x \mathrm{~d} t .
$$

Just as in the laminar solution, the geostrophic velocity, which is equivalent to a large-scale pressure difference, is required to ensure that $\langle v\rangle=0$. We assume that $u_{\mathrm{g} z}=0$. This assumption is physically plausible because $\overline{p_{y}}$ is dominantly due to a very slight meridional tilt of the free surface of the fluid. According to the hydrostatic relation, this tilt produces a meridional pressure gradient which is independent of depth.

We obtain a lower bound on $\left\langle\|\nabla \boldsymbol{u}\|^{2}\right\rangle$ by forming the linear combination:

$$
(4.1)+\alpha(4.2)-G\left\langle\Lambda_{z}(z)(4.3)\right\rangle+G\left\langle\Omega_{z}(z)(4.4)\right\rangle .
$$

This combination imposes the global energy constraint through $\alpha$, and independently the two momentum constraints at every height. Optimization over $\alpha, \Lambda$ and $\Omega$ is therefore analogous to the improvement of the original $\mathrm{CDH}$ method proposed initially by Nicodemus et al. (1997a) in the context of plane Couette flow. In this linear combination, $\alpha$ is a constant and $\Omega(z)$ and $\Lambda(z)$ are arbitrary functions satisfying the homogeneous boundary conditions

$$
\Omega_{z}(-1)=\Omega(-1)=0, \quad \Lambda(0)=\Omega(0)=0 .
$$

In the linear combination (4.6), we use integration by parts to take a $z$-derivative from $\bar{u}_{z z}$ and $\bar{v}_{z z}$. The homogeneous boundary conditions on $\Lambda$ and $\Omega$ ensure that the terms which fall outside integrals vanish. A crucial intermediate step follows from the observation that

$$
\begin{aligned}
\left\langle\Lambda_{z} \bar{u}_{z z}\right\rangle & =\left[\Lambda_{z} \bar{u}_{z}\right]_{-1}^{0}-\left\langle\Lambda_{z z} \bar{u}_{z}\right\rangle, \\
& =G\left[\Lambda_{z}\right]_{-1}^{0}-\left\langle\Lambda_{z z} \bar{u}_{z}\right\rangle, \\
& =G\left\langle\Lambda_{z z}\right\rangle-\left\langle\Lambda_{z z} \bar{u}_{z}\right\rangle
\end{aligned}
$$

In $(4.8 b)$ we have used the identity $\bar{u}_{z}(-1)=\bar{u}_{z}(0)=G$. This identity, amounting to global zonal momentum balance, is obtained by integrating the averaged zonal momentum equation over $z$ and using $\int_{-1}^{0} \bar{v} \mathrm{~d} z=0$. Notice also that the geostrophic 
velocity disappears because $\left\langle\Omega_{z} u_{g}\right\rangle=-\left\langle\Omega u_{g z}\right\rangle=0$, provided $u_{\mathrm{g}}$ is independent of $z$ throughout the channel, showing how crucial this assumption is to our derivation.

Following the integration by parts, one can write the dissipation as

$$
\begin{aligned}
(1+\alpha)\left\langle\|\nabla \boldsymbol{u}\|^{2}\right\rangle= & \left\langle\left[\bar{u}_{z}+\frac{1}{2} G\left(\alpha-\Lambda_{z z}-E^{-1} \Omega\right)\right]^{2}\right\rangle-\frac{1}{4} G^{2}\left\langle\left(\alpha-\Lambda_{z z}-E^{-1} \Omega\right)^{2}\right\rangle \\
& +\left\langle\left[\bar{v}_{z}-\frac{1}{2} G\left(E^{-1} \Lambda-\Omega_{z z}\right)\right]^{2}\right\rangle-\frac{1}{4} G^{2}\left\langle\left(E^{-1} \Lambda-\Omega_{z z}\right)^{2}\right\rangle \\
& +G^{2}\left\langle\Lambda_{z z}\right\rangle+\mathscr{H},
\end{aligned}
$$

where $\mathscr{H}\left[\boldsymbol{u}^{\prime}, \Lambda, \Omega\right]$ is the functional

$$
\mathscr{H}\left[\boldsymbol{u}^{\prime}, \Lambda, \Omega\right]=\left\langle\left\|\nabla \boldsymbol{u}^{\prime}\right\|^{2}\right\rangle+G\left\langle u^{\prime} w^{\prime} \Lambda_{z z}\right\rangle-G\left\langle v^{\prime} w^{\prime} \Omega_{z z}\right\rangle .
$$

The identity in (4.9) expresses the non-dimensional mechanical energy dissipation $\left\langle\|\nabla \boldsymbol{u}\|^{2}\right\rangle$ in terms of the production $G\left\langle\bar{u}_{z}\right\rangle$ and a projection of the mean momentum balance onto the functions $\Omega(z)$ and $\Lambda(z)$. The 'spectral constraint', analogous to that of the $\mathrm{CDH}$ method, is the requirement that

$$
0 \leqslant \mathscr{H}\left[\boldsymbol{u}^{\prime}, \Lambda, \Omega\right]
$$

for all incompressible velocity fields $\boldsymbol{u}^{\prime}$ with homogeneous boundary conditions. From inspection of (4.10), and by analogy with the energy stability of the laminar solution discussed in $\S 3$, the spectral constraint corresponds to the requirement that the flow with mean velocity shear $\boldsymbol{U}_{z}=\left(\Lambda_{z z},-\Omega_{z z}, 0\right)$ is energy stable. Therefore, the spectral constraint is an expression of energy stability for a notional flow determined by the Lagrange multipliers, $\Omega(z)$ and $\Lambda(z)$, imposing the horizontally averaged momentum balance. Notice that the boundary conditions imposed in (4.7) are not those of the actual flow. This is a difference between our notional flow and the background flow of the $\mathrm{CDH}$ method, which also acts as the Lagrange multiplier which imposes horizontally averaged momentum. We proceed by assuming that it is possible to find $\Omega(z)$ and $\Lambda(z)$ so that the spectral constraint (4.11) is satisfied.

Now we return to (4.9) and let $\aleph \equiv 1+\alpha$. Since $\alpha$ is a general Lagrange multiplier, we need to treat the cases where $\aleph$ is positive and negative separately. It is thus convenient to repose (4.9) as

$$
\begin{array}{r}
\left\langle\|\nabla \boldsymbol{u}\|^{2}\right\rangle=\aleph^{-1}\left\langle\left[\bar{u}_{z}+\frac{1}{2} G\left(\alpha-\Lambda_{z z}-E^{-1} \Omega\right)\right]^{2}+\left[\bar{v}_{z}-\frac{1}{2} G\left(E^{-1} \Lambda-\Omega_{z z}\right)\right]^{2}+\mathscr{H}\right\rangle \\
+\frac{1}{2} G^{2}\left\langle 1+E^{-1} \Omega+\Lambda_{z z}\right\rangle-\frac{1}{4} G^{2} \aleph-\frac{1}{4} G^{2} \mathscr{F} \aleph^{-1},
\end{array}
$$

where $\mathscr{F}$ is the functional

$$
\mathscr{F}[\Omega, \Lambda] \equiv\left\langle\left(1+E^{-1} \Omega+\Lambda_{z z}\right)^{2}+\left(\Omega_{z z}-E^{-1} \Lambda\right)^{2}-4 \Lambda_{z z}\right\rangle .
$$

Using integration by parts, and the boundary conditions in (4.7) to show that $\left\langle\Omega_{z z} \Lambda\right\rangle=$ $\left\langle\Omega \Lambda_{z z}\right\rangle$, one rewrites $\mathscr{F}$ as

$$
\mathscr{F}[\Omega, \Lambda]=\left\langle\left(1+E^{-1} \Omega-\Lambda_{z z}\right)^{2}+\left(\Omega_{z z}+E^{-1} \Lambda\right)^{2}\right\rangle .
$$

Posing the dissipation as in (4.12), and realizing that $\mathscr{F}$ is non-negative, it is straightforward to generate bounds for either sign of $\aleph=1+\alpha$.

First suppose that $\aleph>0$. Then we obtain a lower bound on the dissipation $\left\langle\|\nabla \boldsymbol{u}\|^{2}\right\rangle$ by dropping the first positive term on the right-hand side of (4.12):

$$
G^{-2}\left\langle\|\nabla \boldsymbol{u}\|^{2}\right\rangle \geqslant \frac{1}{2}\left\langle 1+E^{-1} \Omega+\Lambda_{z z}\right\rangle-\frac{1}{4} \aleph-\frac{1}{4} \mathscr{F} \aleph^{-1} .
$$


Viewed as a function of $\aleph$, the right-hand side (4.15) has a maximum at $\aleph=\sqrt{\mathscr{F}}$. Substituting $\aleph=\sqrt{\mathscr{F}}$ into (4.15) gives the best lower bound available by optimizing over $\aleph>0$ :

$G^{-2}\left\langle\|\nabla \boldsymbol{u}\|^{2}\right\rangle \geqslant \frac{1}{2}\left[\left\langle 1+E^{-1} \Omega+\Lambda_{z z}\right\rangle-\sqrt{\left\langle\left(1+E^{-1} \Omega-\Lambda_{z z}\right)^{2}+\left(\Omega_{z z}+E^{-1} \Lambda\right)^{2}\right\rangle}\right]$.

Now return to (4.12) and suppose that $\aleph<0$. Dropping the first term on the righthand side of (4.12) now produces an upper bound. Thus the inequalities are reversed in (4.15) and (4.16). The other difference is that the $\aleph$-optimization problem now results in a smallest upper bound at $\aleph=-\sqrt{\mathscr{F}}$, and so the sign in front of the square root in (4.16) is now a plus.

We summarize our dual bounds as

$$
\mathscr{B}_{-}[\Lambda, \Omega] \leqslant G^{-2}\left\langle\|\nabla \boldsymbol{u}\|^{2}\right\rangle \leqslant \mathscr{B}_{+}[\Lambda, \Omega],
$$

where the bounding functionals, $\mathscr{B}_{ \pm}[\Lambda, \Omega]$, are

$$
\mathscr{B}_{ \pm}[\Lambda, \Omega] \equiv \frac{1}{2}\left[1+E^{-1}\langle\Omega\rangle+\left\langle\Lambda_{z z}\right\rangle \pm \sqrt{\left\langle\left(1+E^{-1} \Omega-\Lambda_{z z}\right)^{2}+\left(\Omega_{z z}+E^{-1} \Lambda\right)^{2}\right\rangle}\right] .
$$

In these expressions, $\Omega(z)$ and $\Lambda(z)$ are any two functions which satisfy the boundary conditions in (4.7) and the spectral constraint in (4.11).

\section{The pseudo-laminar solution}

Further progress depends on obtaining extremes of the right-hand side of (4.18) by choosing $\Lambda(z)$ and $\Omega(z)$. This choice is restricted by the boundary conditions in (4.7) and the spectral constraint $\mathscr{H} \geqslant 0$. As noted in our discussion of (4.11), the spectral constraint corresponds to demanding that the flow with mean velocity shear $\boldsymbol{U}_{z}=\left(\Lambda_{z z},-\Omega_{z z}, 0\right)$ be energy stable. Therefore, loosely, it is reasonable to think that $\Lambda_{z}$ is analogous to $\bar{u}$ and $-\Omega_{z}$ is analogous to $\bar{v}$. This motivates us to write

$$
\Lambda_{z}=\beta\left(\tilde{U}-\tilde{U}_{\mathrm{g}}\right), \quad \Omega_{z}=-\beta \tilde{V},
$$

where $\tilde{U}(z)$ and $\tilde{V}(z)$ are obtained by solving the 'pseudo-laminar' problem

$$
\begin{aligned}
-\tilde{V} & =\tilde{E} \tilde{U}_{z z}, \\
\tilde{U}-\tilde{U}_{\mathrm{g}} & =\tilde{E} \tilde{V}_{z z},
\end{aligned}
$$

with the boundary conditions

$$
\tilde{U}(-1)=\tilde{V}(-1)=0, \quad \tilde{V}_{z}(0)=0, \quad \tilde{U}_{z}(0)=1 .
$$

Notice that the final boundary condition above sets the amplitude of the pseudolaminar velocity fields $(\tilde{U}, \tilde{V})$. We refer to $(\tilde{U}, \tilde{V})$ as the pseudo-laminar problem because the parameter $\tilde{E}$ is generally not equal to $E$, and indeed we find that the best bounds we can construct using $(\tilde{U}, \tilde{V})$ are obtained by making $\tilde{E}$ a function of $G$.

The best bounds are produced by setting the amplitude parameter $\beta$ in (5.1) so that the spectral constraint $(\mathscr{H} \geqslant 0)$ is marginally satisfied:

$$
\tilde{\eta} \equiv(2 \tilde{E})^{-1 / 2}, \quad \beta=\frac{G_{\text {es }}(\tilde{\eta})}{G} .
$$

Above, $G_{\text {es }}(\tilde{\eta})$ is the energy stability function shown in figure $3(a)$. 
The advantage of the pseudo-laminar problem is that using (5.1), (5.2) and (5.3), and some integration by parts, one can prove the following identities:

$$
\begin{aligned}
\left\langle\Omega_{z z}^{2}+\Lambda_{z z}^{2}\right\rangle & =\beta^{2} \tilde{U}_{0}, \\
\left\langle\Omega^{2}+\Lambda^{2}\right\rangle & =\beta^{2} \tilde{E}^{2}\left(1-\tilde{U}_{0}\right), \\
\langle\Omega\rangle & =\beta \tilde{E}\left(\tilde{U}_{0}-1\right) .
\end{aligned}
$$

$\tilde{U}_{\mathrm{g}}(\tilde{\eta})$ and $\tilde{U}_{0}(\tilde{\eta})$ have the same functionality as $U_{\mathrm{g}}(\eta)$ and $U_{0}(\eta)$ defined in (3.5) and (3.6). With the results in (5.2), the dual bound in (4.18) becomes

$$
\begin{aligned}
\mathscr{B}_{ \pm}\left(G, \tilde{E} ; R o_{\star}\right)= & \frac{1}{2}\left[1-\beta \gamma+\beta(1+\gamma) \tilde{U}_{0}\right. \\
& \left. \pm \sqrt{1-2 \beta \gamma+\beta^{2} \gamma^{2}+\beta^{2} \tilde{U}_{0}\left(1-\gamma^{2}\right)-2 \beta \tilde{U}_{0}(1-\gamma)}\right]
\end{aligned}
$$

where $\gamma \equiv \tilde{E} / E=\tilde{E} \sqrt{G} / R o_{\star}$, and $\beta(\tilde{E}, G)$ is determined by (5.4). We use the friction Rossby number $R o_{\star} \equiv \sqrt{G} E$ in preference to $E$ because, as explained in the discussion surrounding (2.4), the inviscid limit is $G \rightarrow \infty$ with $R o_{\star}$ fixed.

For orientation, a simple special case is a laminar flow that is energy stable. $\dagger$ Then we can pick $\beta=\gamma=1$ and (5.6) collapses to

$$
\mathscr{B}_{ \pm}=U_{0}(\eta)
$$

where $U_{0}(\eta)$ is defined in (3.6). This is obvious: below energy stability the flow is laminar, and the energy dissipation is uniquely $U_{0}(E)$. In this case the upper and lower bounds coincide on the laminar dissipation as they must.

Once $G$ is increased past the energy stability threshold, $G_{\text {es }}(E)$, the laminar flow is no longer energy stable, and the laminar dissipation is neither an upper nor a lower bound in this framework. In this case, particularly simple bounds are produced by taking $\gamma \equiv \tilde{E} / E=1$ and marginally satisfying the spectral constraint with $\beta$ in (5.4). With $\gamma=1$, (5.6) simplifies considerably so that the dual bound is

$$
1-G^{-1} G_{\mathrm{es}}(\eta)+G^{-1} G_{\mathrm{es}}(\eta) U_{0}(E) \geqslant\left\langle\|\nabla \boldsymbol{u}\|^{2}\right\rangle \geqslant G^{-1} G_{\mathrm{es}}(\eta) U_{0}(E)
$$

In the rapidly rotating limit, with $\eta \equiv(2 E)^{-1 / 2} \gg 1$, we have $G_{\text {es }}(\eta) \approx 5.86 \eta^{2}=$ $2.93 \mathrm{fh}^{2} / \mathrm{v}$ (see the dashed curve in figure $3 a$ ) and $U_{0}(E) \approx 3 E / 2$. Using these approximations, and expressing (5.8) in dimensional variables, we find that

$$
\frac{u_{\star}^{4}}{v}-2.93 f u_{\star}^{2}+6.215 u_{\star}^{2} \sqrt{f v} \geqslant \varepsilon \geqslant 6.215 u_{\star}^{2} \sqrt{f v} .
$$

Close to the rapidly rotating energy stability threshold, the dual bounds in (5.9) tightly bracket $\varepsilon$. However as $v \rightarrow 0$ the bounds diverge: the upper bound asymptotes to the Stokes dissipation and the lower bound approaches zero. To improve this situation we must take advantage of the parameter $\tilde{E}$, i.e. one obtains better bounds with $\gamma \equiv \tilde{E} / E \neq 1$.

With $\gamma \neq 1$ in (5.6), the simplest approach is to fix $R o_{\star}$ and plot $\mathscr{B}_{ \pm}\left(G, \tilde{E} ; R o_{\star}\right)$ in (5.6) as a function of $G$ for various values of $\tilde{E}$. For example, in figure $4, R o_{\star}=0.43$. In this case the flow is energy stable only if $G$ is less than $G_{\text {es }}(0.43)=117.24$. The

$\dagger$ If the laminar solution is energy stable the spectral constraint is ensured by taking $\beta=1$, rather than using (5.4). 


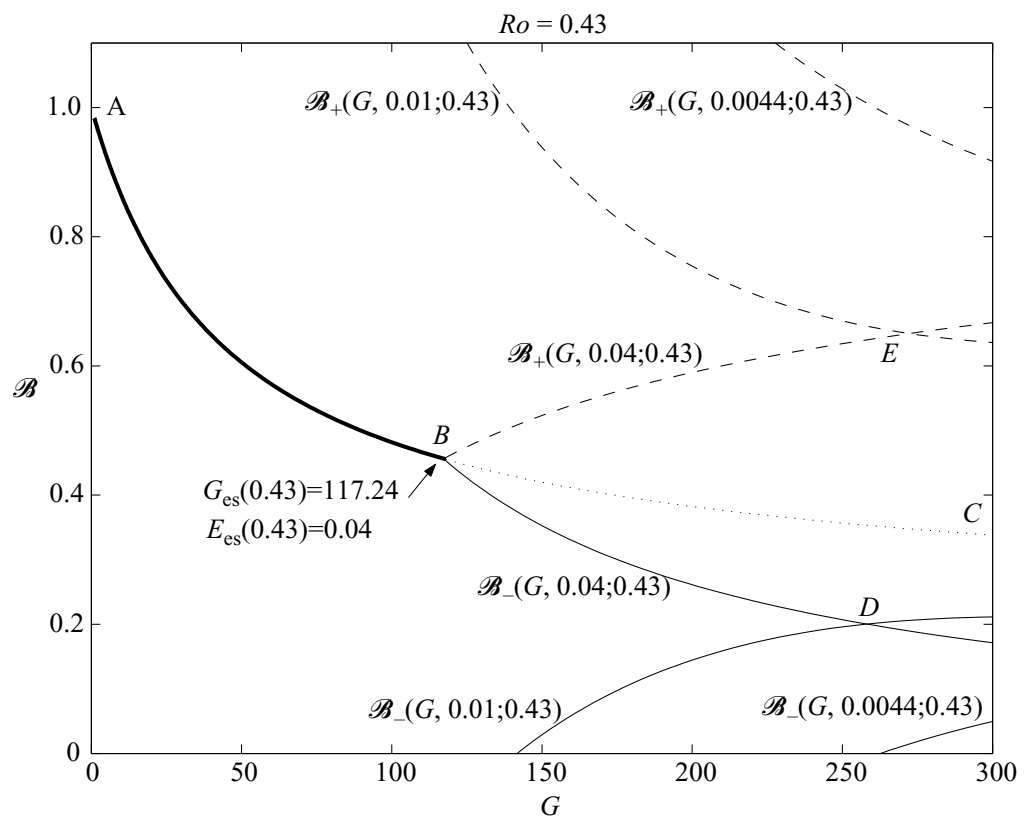

FIGURE 4. In this figure $R o_{\star} \equiv \sqrt{G} E=0.43$, and the rotating laminar dissipation corresponds to the curve $A B C$. The solution is energy stable if $G<G_{\text {es }}=117.24$ (equivalently $E>E_{\mathrm{es}}=0.04$ ). Thus if $G \leqslant G_{\text {es }}=117.24$, then the rotating laminar dissipation is the unique bound (the heavy curve $A B$ ). The dual bounds in (5.12) bifurcate from the laminar dissipation at the energy stability point $B$. If $G>G_{\text {es }}=117.24$ the actual dissipation is between $\mathscr{B}_{+}(G, 0.04 ; 0.43)$ and $\mathscr{B}_{-}(G, 0.04 ; 0.43)$. However by taking $\tilde{E}<E_{\text {es }}=0.04$ (i.e. $\left.\gamma \neq 1\right)$ one can obtain better bounds, e.g. at the points $D$ and $E$ improved bounds are obtained using values of $\tilde{E}$ other than $R o_{\star} / \sqrt{G_{\mathrm{es}}}$.

dissipation of the laminar solution is the curve $A B C$ in figure 4, which is obtained from the function

$$
\mathscr{B}_{\mathrm{L}}\left(G ; R o_{\star}\right) \equiv U_{0}(\eta),
$$

with $\eta \equiv(2 E)^{-1 / 2}=2^{-1 / 2} G^{1 / 4} R o_{\star}^{-1 / 2}$. In figure 4 the curve $A B C$ is

$$
A B C=\mathscr{B}_{\mathrm{L}}\left(G ; R o_{\star}=0.43\right) .
$$

The curve $A B C$ lies between the upper and lower bounds $B E$ and $B D$, which correspond to

$$
B E=\mathscr{B}_{+}\left(G, \frac{R o_{\star}}{\sqrt{G_{\mathrm{es}}}} ; R o_{\star}=0.43\right), \quad \text { and } \quad B D=\mathscr{B}_{-}\left(G, \frac{R o_{\star}}{\sqrt{G_{\mathrm{es}}}} ; R o_{\star}=0.43\right),
$$

i.e. to $\tilde{E}=R o_{\star} / \sqrt{G_{\text {es }}}$, or equivalently $\gamma=\beta^{-1 / 2} . B E$ and $B D$ bifurcate from the laminar dissipation at the energy stability point $B$. Thus, close to $B$, the dissipation is tightly constrained by $B E$ and $B D$. But away from $B$ one obtains better bounds using values of $\tilde{E}$ other than $\tilde{E}=R o_{\star} / \sqrt{G_{\text {es }}}$, e.g. in figure 4 the curve $\mathscr{B}_{-}(G, 0.01 ; 0.43)$ is a better lower bound than $B D$ once $G \geqslant 265$.

By plotting $\mathscr{B}_{ \pm}\left(G, \tilde{E} ; R o_{\star}\right)$ as a function of $G$, with $R o_{\star}$ fixed, and with densely spaced values of $\tilde{E}$, one obtains the optimal (as a function of $\tilde{E}$ ) bounds as upper 

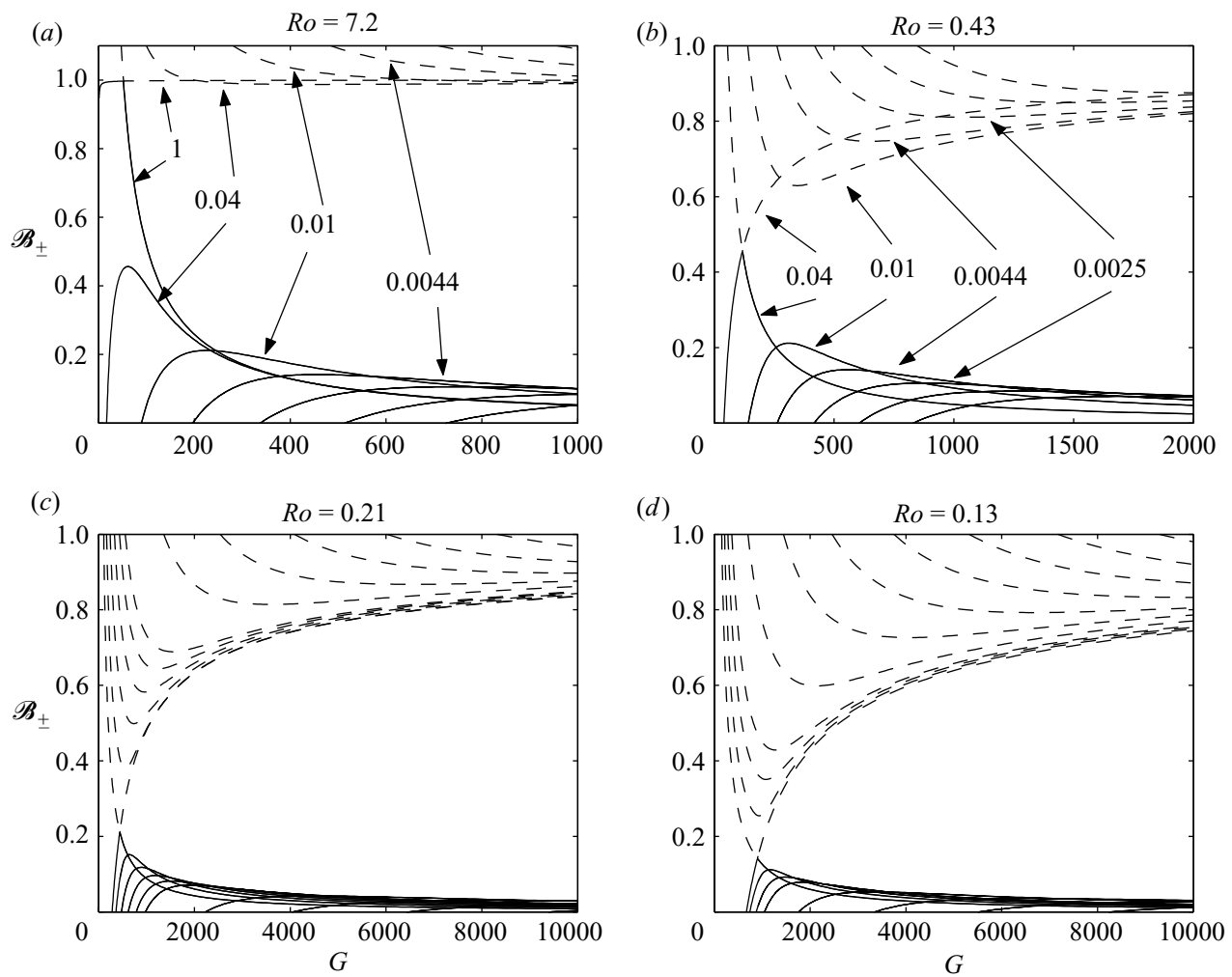

FIGURE 5. Plots of the dual bounds $\mathscr{B}_{ \pm}$in (5.6) associated with four choices of $R o_{\star}:(a) R o_{\star}=$ 7.2; (b) $R o_{\star}=0.43 ;$ (c) $R o_{\star}=0.21 ;(d) R o_{\star}=0.13$. In $(a)$ and $(b)$ the values of $\tilde{E}$ are indicated. The $\tilde{E}$-optimized bounds are obtained as the envelope of the family of curves generated by continuously varying $\tilde{E}$. As $G$ increases, curves associated with smaller and smaller values of $\tilde{E}$ become the best bound.

and lower envelopes (see figure 5). Notice that as $G$ increases in figure 5, curves corresponding to smaller and smaller values of $\tilde{E}$ produce the optimal bound.

Close to the energy stability threshold, and for strongly rotating flows, $\mathscr{B}_{+} \ll 1$. As $G \rightarrow \infty, \mathscr{B}_{+} \rightarrow 1$ from below. Therefore, $\mathscr{B}_{+}$associated with the pseudo-laminar profiles always provides a better upper bound than the Stokes flow. As we can see in figure 5, the energy stability point $G_{\text {es }}$ is a function of $R o_{\star}$. When $R o_{\star}$ decreases, $G_{\text {es }}\left(R o_{\star}\right)$ increases, i.e. as the flow rotates faster, the laminar flow is more energy stable. The relation between $R o_{\star}$ and $G_{\text {es }}\left(R o_{\star}\right)$ is also shown in table 1. Finally, one might notice that for large values of $R o_{\star}$ (figure $5 a$ ), rotation is very weak, and the bound is very similar to the non-rotating bound in TCY04.

When $G$ is very large, asymptotic results can be derived by analytically solving the envelope construction in figure 5. After the discussion in the Appendix, we find that for the upper bound,

$$
\mathscr{B}_{+} \rightarrow \mathscr{B}_{+\infty} \equiv 1-\frac{5.86 E}{2 R o_{\star}^{2}}
$$

or, dimensionally, $\varepsilon_{\max } \geqslant \varepsilon$ where

$$
\varepsilon_{\max } \approx u_{\star}^{4} / \nu-2.93 u_{\star}^{2} f .
$$


(a)

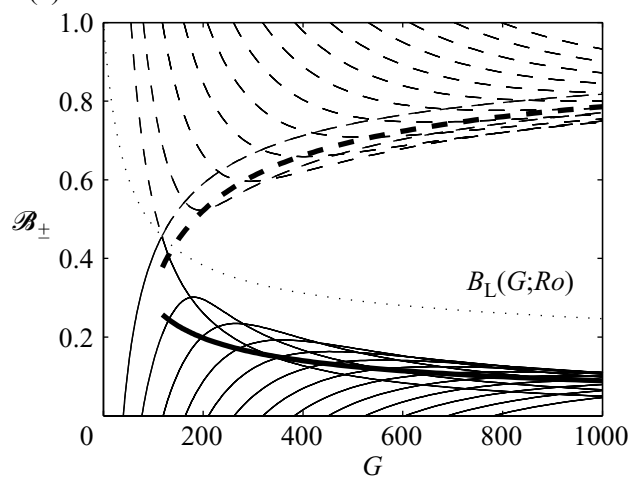

(b)

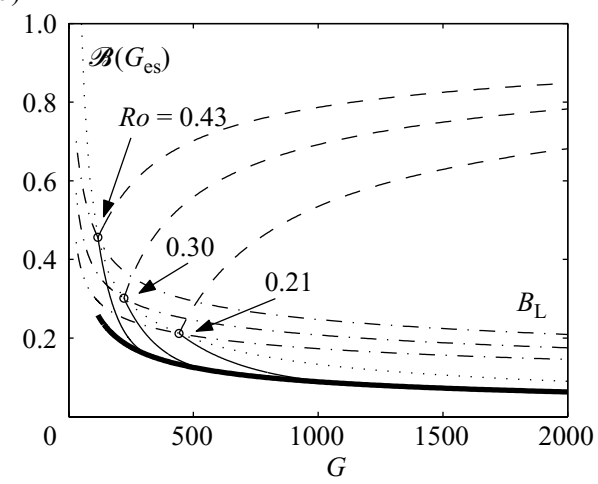

Figure 6. (a) Plots of the upper bound $\mathscr{B}_{+}$as defined in (5.6) (envelope of the thin dashed lines, each of which corresponds to a different value of $\tilde{E}$ in $(5.2)$ ), the lower bound $\mathscr{B}_{-}$ (envelope of the thin solid lines), and the dissipation associated with the laminar solution $\mathscr{B}_{\mathrm{L}}$ defined in (5.7) (the dotted line) at the choice of $R o_{\star}=0.43$. As $G \rightarrow \infty$, the two bounds approach their respective asymptotic limits $\mathscr{B}_{+\infty}$ (defined by $(5.13)$ and plotted with a thick dashed line) and $\mathscr{B}_{-\infty}$ (defined by (5.15) and plotted with a thick solid line). (b) A comprehensive plot of the dual bound $\mathscr{B}_{ \pm}$(plotted as dash-dotted lines below energy stability, where $\mathscr{B}_{+}=\mathscr{B}_{-}$, and as thin solid lines for $\mathscr{B}_{-}$and thin dashed lines for $\mathscr{B}_{+}$above the relevant energy stability point for the particular, labelled value of $R o_{\star}$ ), the asymptotic lower bound $\mathscr{B}_{-\infty}=2.795 G^{-1 / 2}$ (the thick solid line) and the laminar dissipation $\mathscr{B}_{\mathrm{L}}$ (the dash-dotted lines) for different $R o_{\star}$.

This scaling approaches the Stokes dissipation rate in the limit $v \rightarrow 0$. For the lower bound,

$$
\mathscr{B}_{-} \rightarrow \mathscr{B}_{-\infty} \equiv 2.795 G^{-1 / 2},
$$

or, dimensionally, $\varepsilon \geqslant \varepsilon_{\min }$, where

$$
\varepsilon_{\min } \approx 2.795 u_{\star}^{3} / h .
$$

This scaling is independent of both viscosity and rotation in the limit $v \rightarrow 0$.

To illustrate how the upper and lower bound move away from the laminar solution at the laminar energy stability point and approach their asymptotic limits, we have plotted $\mathscr{B}_{ \pm}$, together with the laminar dissipation $\mathscr{B}_{\mathrm{L}}=U_{0}(E)$ for $R o_{\star}=0.43$ in figure $6(a)$. The bounds move away from the laminar solution at the energy stability point, and converge, as $G \rightarrow \infty$, to the asymptotic upper and lower bounds $\mathscr{B}_{+\infty}$ (shown with a thick dashed line) and $\mathscr{B}_{-\infty}$ (shown with a thick solid line) defined by (5.13) and (5.15) respectively.

In figure $6(b)$, we show in detail how, for different choices of $R o_{\star}$, the lower bound $\mathscr{B}_{-}$moves away from the laminar bound $\mathscr{B}_{\mathrm{L}}$ at the relevant energy stability point (which naturally varies with $R o_{\star}$ ). The dotted line marks the locus of energy stability points for varying $R o_{\star}$. The dash-dotted lines show the dissipation associated with the laminar solution after it loses energy stability for fixed values of $R o_{\star}$ for each curve. At a particular value of $R o_{\star}$, the upper bound (plotted with a dashed line) increases above this dissipation, and approaches its asymptotic limit $\mathscr{B}_{+\infty}$, defined by (5.13). Conversely, the lower bound (plotted with a thin solid line) then approaches the asymptotic lower bound $\mathscr{B}_{-\infty}$, defined by (5.15) (and plotted with a thick solid line). As is apparent from the figure, not only does the bifurcation away from the rotating laminar solution occur for higher and higher $G$ as $R o_{\star}$ decreases, but also the approach to the asymptotic bound $\mathscr{B}_{-\infty}$ takes longer and longer as $R o_{\star}$ decreases. For 
intermediate values of $G$ the approach of the lower bound to its asymptote depends strongly on the particular value of $R o_{\star}$, and hence the rotation rate, as fixing $R o_{\star}$ implies that the Ekman number decreases as $G^{1 / 2}$ increases.

\section{Conclusions}

We have constructed rigorous dual bounds on the mechanical energy dissipation rate for surface-stress-driven rotational flow, subject to certain assumptions, in particular that the geostrophic velocity $u_{\mathrm{g}}$ defined in (4.5) is independent of depth throughout the channel. To handle the complications associated with the Coriolis force, it is necessary to include integral constraints which capture both components of the mean horizontal momentum balance. Another particularly important aspect of the method is the marginal satisfaction of the appropriate spectral constraint (e.g. as in our determination of the parameter $\beta$ in (5.4)). This is a point of similarity with PK03 and TCY04, where the optimal bounds have been obtained by solving the full EulerLagrange equations, which ensures that the spectral constraint is marginally satisfied. The approach used here is simpler than solving the full variational problem, but retains this key aspect.

In the inviscid limit our dual bounds in (5.14) and (5.16) are

$$
u_{\star}^{4} / v-2.93 u_{\star}^{2} f \geqslant \varepsilon \geqslant 2.795 u_{\star}^{3} / h
$$

of course, these inequalities are likely to be conservative. If we make the plausible assumption that $\varepsilon$ is independent of $v$ in the inviscid limit, then dimensional considerations imply that

$$
\varepsilon=\frac{u_{\star}^{3}}{h} \mathscr{J}\left(\frac{u_{\star}}{f h}\right),
$$

where $\mathscr{J}$ is a dimensionless function. The lower bound above indicates that $\mathscr{J}$ is greater than 2.795 for all values of $R o_{\star}=u_{\star} /(f h)$, but the method has not yielded more detailed information about the scaling function $\mathscr{J}\left(R o_{\star}\right)$. If the lower bound (5.16) correctly indicates the scaling of the inviscid limit (i.e. that $\mathscr{J}$ is constant) then the following picture of the mean flow emerges. The surface velocity is of order $u_{\star}$, so that the rate of working of the stress is $u_{\star}^{3}$. If the velocity at depth is zero, then the reduction from the surface maximum to $u_{\star}$ to zero occurs in a boundary layer of thickness $v / u_{\star}$. This scenario does not seem realistic because one expects $u_{\star} / f$ to appear as an important length in a turbulent Ekman layer (Gill 1982).

A main complication is that the rotating laminar solution (3.4) differs from the Stokes flow in (2.7). Thus we cannot conclude that the laminar dissipation bounds the true dissipation in either direction except, of course, below the energy stability threshold. When the flow is just past energy stability the dual bounds closely bracket the dissipation and in the case of rapid rotation $\left(R o_{\star} \ll 1\right)$ the upper bound is significantly smaller than, and therefore significantly better than, the upper bound provided by the Stokes flow. Thus the bounds show that the Coriolis parameter $f$ is important directly after bifurcation away from the laminar flow.

As with bounding estimates for simpler flows, such as Couette flow, we must view (6.1) with caution. There is no reason why a statistically stationary flow should be organized so as to make its mechanical energy dissipation rate extreme. And even if the $u_{\star}^{3} / h$ scaling is correct, there is no reason to suppose that the quantitative numerical factor 2.795 is fixed. It is possible that profiles other than the pseudolaminar functions in (5.1) might improve the bounds, although previous experience (see TCY04) suggests that the dimensional scaling dependence of the bound may not 
change. Therefore, it would only be meaningful to carry out numerical simulation (as done recently for the related problem of body-forced plane shear flow with stress-free boundary conditions by Doering, Eckhardt \& Schumacher (2003)) to adequately high Grashof numbers to identify the asymptotic scaling behaviour, with which we could then compare the bounding solutions presented here, and possibly also identify other further constraints that we could use to improve our bounds.

We would like to thank the National Science Foundation for support under the Collaborations in Mathematical Geosciences (CMG) initiative (ATM-0222104).

\section{Appendix. Asymptotics for the 'pseudo-laminar profile'}

When $\tilde{\eta}$ is large (which corresponds to the behaviour as $G$ becomes large) all terms involving $\beta$ in the square root in (5.6) are small compared to one, and so we can simplify the bounds to be

$$
\begin{aligned}
& \mathscr{B}_{+}=1-\beta \gamma+\beta \gamma \tilde{U}_{0}+\beta^{2} \gamma^{2} / 4+\beta^{2}\left(1-\gamma^{2}\right) \tilde{U}_{0} / 4, \\
& \mathscr{B}_{-}=\beta \tilde{U}_{0}-\beta^{2} \gamma^{2} / 4-\beta^{2} \tilde{U}_{0}\left(1-\gamma^{2}\right) / 4 .
\end{aligned}
$$

In this asymptotic limit, $\beta G \approx 5.86 \tilde{\eta}^{2}, \tilde{U}_{0} \approx 3 /(2 \tilde{\eta})$, and remembering that $\gamma \equiv$ $\left(2 \tilde{\eta}^{2} E\right)^{-1},(\mathrm{~A} 1)$ becomes

$$
\begin{aligned}
& \mathscr{B}_{+}=1-\frac{5.86}{2 G E}+\frac{3 \times 5.86}{4 G E \tilde{\eta}}+\frac{5.86^{2}}{16 G^{2} E^{2}}+\frac{3 \times 5.86^{2} \tilde{\eta}^{3}}{8 G^{2}}-\frac{3 \times 5.86^{2}}{32 G^{2} E^{2} \tilde{\eta}}, \\
& \mathscr{B}_{-}=\frac{3 \times 5.86 \tilde{\eta}}{2 G}-\frac{5.86^{2}}{16 G^{2} E^{2}}-\frac{3 \times 5.86^{2} \tilde{\eta}^{3}}{8 G^{2}}+\frac{3 \times 5.86^{2}}{32 G^{2} E^{2} \tilde{\eta}} .
\end{aligned}
$$

To optimize both bounds with respect to $\tilde{\eta}$, we differentiate with respect to $\eta$ and so obtain for the upper bound

$$
\tilde{\eta}^{4}-\frac{2 G}{3 \times 5.86 E}+\frac{1}{12 E^{2}}=0
$$

and for the lower bound,

$$
\tilde{\eta}^{4}-\frac{4 G \tilde{\eta}^{2}}{3 \times 5.86}+\frac{1}{12 E^{2}}=0 .
$$

Equation (A 3) implies that to optimize the upper bound,

$$
\tilde{\eta}^{4}=\frac{8 G E-5.86}{12 \times 5.86 E^{2}} .
$$

With $R o_{\star}^{2}=G E^{2}$ fixed such that $E \rightarrow 0$ and $G \rightarrow \infty$, in the asymptotic limit $G E=R o_{\star}^{2} / E \gg 1$, and so

$$
\tilde{\eta}^{4}=\frac{2 G^{3 / 2}}{3 \times 5.86 R o_{\star}} .
$$

Surprisingly, this result suggests that the leading-order term involving $G$ in (A $2 a)$ is $5.86 /(2 G E)$, which is independent of $\tilde{\eta}$ :

$$
\mathscr{B}_{+} \rightarrow \mathscr{B}_{+\infty} \equiv 1-\frac{5.86 E}{2 R o_{\star}^{2}},
$$

or, dimensionally, $\varepsilon_{\max } \geqslant \varepsilon$ where

$$
\varepsilon_{\max } \approx u_{\star}^{4} / v-2.93 u_{\star}^{2} f .
$$


In the asymptotic limit, $v \ll 1$, and so $\varepsilon_{\max }$ approaches the Stokes dissipation rate $u_{\star}^{4} / \nu$.

Now we look at (A 4) which optimizes the lower bound. The solution for $\tilde{\eta}$ is

$$
\tilde{\eta}^{2}=\frac{2 G}{3 \times 5.86}\left(1 \pm \sqrt{1-\frac{3 \times 5.86^{2}}{16 G^{2} E^{2}}}\right) .
$$

Since we are looking at a limit where the (true) laminar solution is definitely not energy stable, and $R o_{\star}^{2}=G E^{2}$ is fixed, $G^{2} E^{2} \gg 1$, so $\tilde{\eta}^{2}$ takes two possible values:

$$
\tilde{\eta}_{a}^{2} \approx \frac{4 G}{3 \times 5.86} \text { and } \tilde{\eta}_{b}^{2} \approx \frac{5.86}{16 R o_{\star}^{2}} .
$$

To identify the extrema, we differentiate (A $2 b$ ) twice with respect to $\tilde{\eta}$ to obtain

$$
\frac{\partial^{2} \mathscr{B}_{-}}{\partial \tilde{\eta}^{2}}=\frac{3 \times 5.86^{2}\left(1-12 E^{2} \tilde{\eta}^{4}\right)}{16 G^{2} E^{2} \tilde{\eta}^{3}} .
$$

Therefore, $\tilde{\eta}_{a}$ corresponds to a maximum, which is the correct choice for generating a lower bound, whereas $\tilde{\eta}_{b}$ corresponds to a minimum, and so can be ignored.

The asymptotic lower bound associated with $\tilde{\eta}_{a}$ is

$$
\mathscr{B}_{-}=\frac{2 \sqrt{3 \times 5.86}}{3 G^{1 / 2}}-\frac{5.86^{2}}{16 G R o_{\star}^{2}}+\frac{3^{3 / 2} \times 5.86^{5 / 2}}{64 G^{3 / 2} R o_{\star}^{2}},
$$

and to leading order as $G \rightarrow \infty$ at fixed $R o_{\star}$,

$$
\mathscr{B}_{-} \approx \mathscr{B}_{-\infty}=2.795 G^{-1 / 2} \text {. }
$$

Dimensionally, this result means that

$$
\varepsilon \geqslant 2.795 u_{\star}^{3} / h
$$

a scaling that is independent of both viscosity and rotation. This is clearly an improvement on the simple result (5.9) obtained from choosing $\gamma=1$.

Even though our simple approach does not solve the full Euler-Lagrange equations using the $\mathrm{CDH}$ method, and so we do not have a direct means of determining the extremal flow fields, we can still estimate the structure of the mean flow by some scaling arguments. If the lower bound obtained here yields the correct scaling, then the zonal surface velocity $u(0)$ decelerates greatly from its laminar value $O\left(G E^{1 / 2}\right)$ to $O\left(G^{1 / 2}\right)$, or dimensionally, from $O\left(u_{\star}^{2} / \sqrt{v f}\right)$ to $O\left(u_{\star}\right)$. We know that the fixed surface stress requires the shear at the boundaries to be $G$. Therefore, in order to produce the required energy dissipation, the mean flow has to develop boundary layers of thickness $O\left(G^{-1 / 2}\right)$, the same order as the Lagrange multipliers. Dimensionally, the boundary layers of the mean flow are $O\left(v / u_{\star}\right)$, considerably sharper than those of the laminar solutions, which have boundary layer thickness of $O\left(\left[v h / u_{\star}\right]^{1 / 2}\right)$.

\section{REFERENCES}

Boyd, J. P. 2001 Chebyshev and Fourier Spectral Methods, 2nd edn. Dover.

Constantin, P. \& Doering, C. R. 1995 Variational bounds on energy dissipation in incompressible flows: II. Channel flow. Phys. Rev. E 51, 3192-3198.

Constantin, P., Hallstrom, C. \& PutKaradze V. 1999 Heat transport in rotating convection. Physica D 125, 275-284.

Constantin, P., Hallstrom, C. \& Poutkaradze V. 2001 Logarithmic bounds for infinite Prandtl number rotating convection. J. Math. Phys. 42, 773-783. 
Doering, C. R. \& Constantin, P. 1992 Energy dissipation in shear driven turbulence. Phys. Rev. Lett. 69, 1648-1651.

Doering, C. R. \& Constantin, P. 1994 Variational bounds on energy dissipation in incompressible flows: Shear flow. Phys. Rev. E 49, 4087-4099.

Doering, C. R. \& Constantin, P. 1996 Variational bounds on energy dissipation in incompressible flows: III. Convection. Phys. Rev. E 53, 5957-5981.

Doering, C. R., Eckhardt, B. \& Schumacher, J. 2003 Energy dissipation in body-forced plane shear flow. J. Fluid Mech. 494, 275-284.

Drazin, P. G. \& ReID, W. H. 1981 Hydrodyamic Stability. Cambridge University Press.

Gill, A. E. 1982 Atmosphere-Ocean Dynamics. Academic.

Hopf, E. 1941 Ein allgemeiner endlichkeitssatz der hydrodynamik. Mathematische Annalen 117, 764-775.

JosePH, D. D. 1976 Stability of Fuild Motions I. Springer.

Nicodemus, R., Grossmann, S. \& Holthaus, M. 1997 a Improved variational principle for bounds on energy dissipation in turbulent shear flow. Physica D 101, 178-190.

Nicodemus, R., Grossmann, S. \& Holthaus, M. 1997b Variational bound on energy dissipation in plane Couette flow. Phys. Rev. E 56, 6774-6786.

Nicodemus, R., Grossmann, S. \& Holthaus, M. 1998a The background flow method. Part 1. Constructive approach to bounds on energy dissipation. J. Fluid Mech. 363, 281-300.

Nicodemus, R., Grossmann, S. \& Holthaus, M. $1998 b$ The background flow method. Part 2. Asymptotic theory of dissipation bounds. J. Fluid Mech. 363, 301-323.

Pedlosky, J. 1987 Geophysical Fluid Dynamics. Springer.

Plasting, S. C. \& Kerswell, R. R. 2003 Improved upper bound on the energy dissipation rate in plane Couette flow: The full solution to Busse's problem and the Constantin-Doering-Hopf problem with one-dimensional background field. J. Fluid Mech. 477, 363-379 (referred to herein as PK03).

Tang, W., Caulfield C. P. \& Young, W. R. 2004 Bounds on dissipation in stress-driven flow. J. Fluid Mech. 510, 333-352 (referred to herein as TCY04).

Vitanov, N. K. 2001 Upper bounds on the convective heat transport in a rotating fluid layer of infinite Prandtl number: Case of large Taylor numbers. Euro. Phys. J. B 23, 249-266.

Vitanov, N. K. 2003 Convective heat transport in a rotating fluid layer of infinite Prandtl number: Optimum fields and upper bounds on Nusselt number. Phys. Rev. E 67, Art. No. 026322.

Vitanov, N. K. \& Busse, F. H. 2001 Bounds on the convective heat transport in a rotating layer. Phys. Rev. E 63, Art. No. 016303.

YAN, X. D. 2004 On limits to convective heat transport at infinite Prandtl number with or without rotation. J. Math. Phys. 45, 2718-2743. 